\title{
Optical inclinometer based on a single long-period fiber grating combined with a fused taper
}

\author{
O. Frazão \\ INESC-Porto, Rua do Campo Alegre, 687, 4169-007 Porto, Portugal \\ R. Falate \\ INESC-Porto, Rua do Campo Alegre, 687, 4169-007 Porto, Portugal, and Universidade Tecnológica Federal do \\ Paraná, Avenida Sete de Setembro, 3165, 80230-901 Curitiba, Brazil \\ J. L. Fabris \\ Universidade Tecnológica Federal do Paraná, Avenida Sete de Setembro, 3165, 80230-901 Curitiba, Brazil \\ J. L. Santos \\ INESC-Porto, Rua do Campo Alegre, 687, 4169-007 Porto, Portugal, and Departamento de Física, Faculdade de \\ Ciências da Universidade do Porto, Rua do Campo Alegre, 687, 4169-007 Porto, Portugal \\ L. A. Ferreira and F. M. Araújo \\ INESC-Porto, Rua do Campo Alegre, 687, 4169-007 Porto, Portugal
}

Received May 24, 2006; revised July 14, 2006; accepted July 21, 2006; posted August 8, 2006 (Doc. ID 71340); published September 25, 2006

\begin{abstract}
A new concept to measure rotation angles based on a fiber-optic modal Mach-Zehnder interferometer is demonstrated by using a nonadiabatic taper cascaded with a long-period fiber grating. Information about the magnitude of the rotation angle can be obtained from the measurement of the interference pattern visibility, and under certain conditions it is also possible to obtain the sign of the rotation angle from the induced phase variation in the fiber interferometer. (C) 2006 Optical Society of America
\end{abstract}

OCIS codes: $050.2770,060.2370$.

A long-period fiber grating (LPG) is an optical device that couples the forward-propagating guided mode to forward-propagating cladding modes of a single-mode optical fiber, leaving a set of attenuation bands in the transmission spectrum. ${ }^{1}$ This forward coupling is obtained by a periodic refractive index modulation of a hundred micrometers along the core of the optical fiber. LPGs turned out to be very valuable in both optical communications and fiber sensing. For the latter, they prove to be effective sensing elements of different measurands, such as temperature, strain, transverse load, curvature, pressure, and refractive index. $^{2}$

LPGs can also be used to implement versatile fibermode interferometers with enhanced parameter sensitivity. This is done either by cascading two similar LPGs within a certain fiber length [Fig. 1(a)], resulting in a single-fiber Mach-Zehnder configuration, ${ }^{3-5}$ or by using a single LPG and a mirror [Fig. 1(b)], producing a fiber-mode Michelson interferometer. ${ }^{6,7}$ The general principle of operation of both interferometers is illustrated in Figs. 1(a) and 1(b). Light propagates along the optical fiber to the first long-period grating, which couples part of the light to one of the cladding modes. At the cladding, light propagates with an effective refractive index smaller than the one experienced by the light that propagates in the core. In this way, a differential optical path length is accumulated between the two light waves. At the second LPG, a similar fraction of the core light couples into the same cladding mode, being lost after a propagation length much larger than the separation between the two LPGs; reciprocally, a certain amount of the light propagating in the cladding mode is coupled back into the core. Therefore after the second LPG two optical waves with a differential optical path delay propagate in the fiber core, resulting in an interference pattern that has the well-known channeled spectrum structure when broadband light is injected into the input fiber. A quantitative manner to determine the quality or contrast of the fringe pattern interference is calculating the visibility, which is given by $\nu(r)=\left(I_{\max }-I_{\min }\right) /\left(I_{\max }+I_{\min }\right)$, where $I_{\max }$ and $I_{\min }$ are the maximum and adjacent minimum irradiances of the interference fringe, respectively. ${ }^{8}$

To have an interference pattern with good visibility $\left(I_{\min } \approx 0\right)$, two identical LPGs with a peak loss near $3 \mathrm{~dB}$ are required. In fact, the fiber-mode Michelson interferometer described before [Fig. 1(b)] was proposed to overcome such constraint. This interferom-

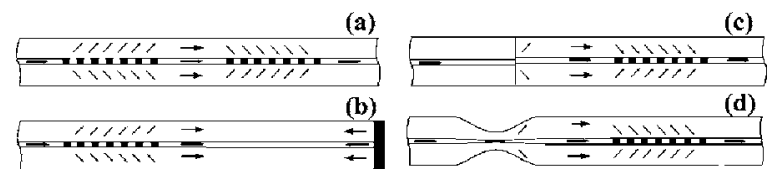

Fig. 1. Optical fiber interferometer based on (a) two similar LPGs, (b) LPG and a mirror, (c) misaligned splice and a LPG, (d) LPG combined with a fused taper. 
(a)

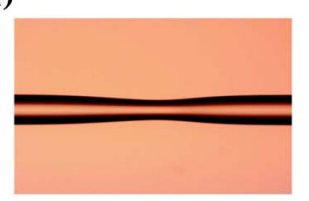

(b)

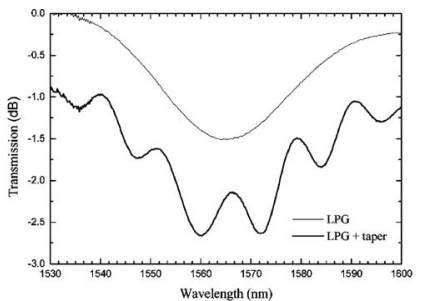

(c)

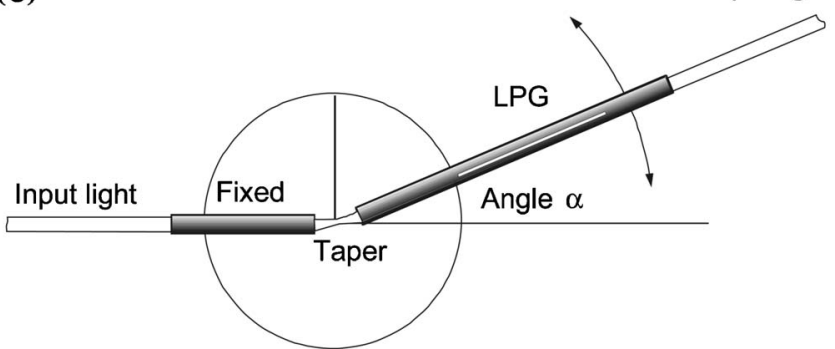

Fig. 2. (Color online) (a) Photograph of the optical taper. (b) Fiber transmission spectrum before and after the fabrication of the fused taper. (c) Inclinometer sensing head.

eter was explored by Lee and $\mathrm{Nishii}^{6}$ to implement a temperature sensor and by Swart $^{7}$ to aim refractive index measurements. However, there are situations where it is necessary to operate in transmission while keeping the requirement of using a single LPG. Dong et al. ${ }^{9}$ reported a configuration where the coupling of light from the core mode to the cladding modes is obtained through a misaligned splicing point as illustrated in Fig. 1(c). This technique is globally effective, but it would be desirable to have an alternative configuration without a core discontinuity and therefore with the potential for an intrinsically smaller insertion loss.

In this Letter we propose a new approach for a fiber-optic modal Mach-Zehnder configuration with a single LPG. It is based on the fabrication of a taper that couples a fraction of the core light to the cladding modes and an LPG placed some distance after the taper that recouples light in the cladding modes into the fiber core. This concept is experimentally validated and applied to the implementation of a fiber-optic inclinometer sensor.

The fiber-optic modal interferometer explored in this work is schematically represented in Fig. 1(d). It consists of a fiber taper followed by a LPG. The nonadiabatic taper was fabricated in a Corning SMF-28 fiber by elongating the fiber during the arc discharge, which was provided by a splicing machine. The fabrication parameters were adjusted to decrease the fiber diameter from 125 to $80 \mu \mathrm{m}$ in the taper waist. The resulting structure, shown in Fig. 2(a), has a total length of $\sim 500 \mu \mathrm{m}$ and an insertion loss of $\sim 1 \mathrm{~dB}$. The purpose of the fiber taper is to expand the core field and consequently to provide some light in the cladding so that the LPG located next to the taper can couple back a number of the cladding modes to the fiber core.

The LPG was written in the same Corning SMF-28 fiber by using the electric-arc technique described by Rego et al. ${ }^{10}$ The LPG used in the experiment had the following fabrication parameters: weight, $5.1 \mathrm{~g}$; pe- riod, $540 \mu \mathrm{m}$; electric current, $9 \mathrm{~mA}$; arc duration, $1 \mathrm{~s}$; number of electric discharges, 40 . The transmission spectrum of the grating has an attenuation peak centered at $1565 \mathrm{~nm}$ with an attenuation loss of $1.5 \mathrm{~dB}$ considering the wavelength range used in the experiments. The fused taper and the center of the LPG are separated by $\sim 80 \mathrm{~mm}$ of optical fiber. Figure 2(b) shows the spectral response of the LPG before and after the fabrication of the optical taper. The channeled spectrum, with a fringe periodicity of $\sim 12 \mathrm{~nm}$, results from the interference of light from the cladding mode coupled back into the fiber core with the fraction that kept propagating in the core. From this result, and considering that the length of the fiber interferometer is $\sim 80 \mathrm{~mm}$, a value of $\sim 2.54 \times 10^{-3}$ can be calculated for the refractive index difference between the core and the cladding mode under consideration. This fiber modal interferometer configuration was applied to develop an inclinometer sensing head, which is shown in Fig. 2(c). As seen from this figure, the axis of rotation goes through the center of the taper. Therefore when angle $\alpha$ in the figure changes, the curvature applied to the taper also changes, affecting the coupling of the core mode to the cladding modes. The fiber after the taper is located inside a capillary tube to ensure that the fiber is kept straight for any value of $\alpha$. For the performed experiment, the angle was changed from $-10^{\circ}$ to $+10^{\circ}$. The variation of $\alpha$ has no effect on the phase of the fiber interferometer, and therefore the wavelength position of the interferometric fringes is kept when the angle changes. This result was expected, since the applied angles do not change the differential optical path length. On the other hand, the taper rotation enhances the coupling of light from the core mode to the cladding modes, resulting in larger amplitudes for the interferometric fringes, i.e., in an increase of the interferometer visibility. This is the basis of the concept that allows one to measure rotation with the proposed configuration. From Fig. 3 , it can be observed that the fringe visibility is approximately an even function of the rotation angle (curves connecting the experimental points are added to guide eyes and are not experimentally verified). Such behavior indicates that it is possible to measure the modulus of the rotation angle with the present

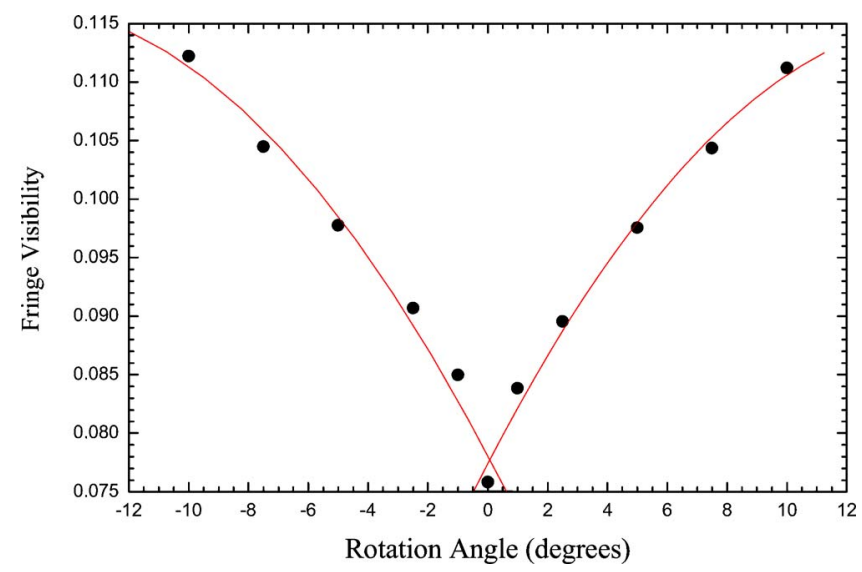

Fig. 3. (Color online) Fringe visibility of the fiber interferometer versus the rotation angle. 
configuration. The nonlinearity of the observed visibility versus the rotation angle is not of too much concern since it can be overcome with proper calibration. On the other hand, fringe visibility is an absolute parameter, i.e., there is no need for any type of referentiation, which is not true with other approaches (for example, removing the LPG and simply monitoring the optical power propagating in the fiber core after the taper as a function of the rotation angle). Indeed, this is an interesting and important feature of this sensing structure.

The symmetric results shown in Fig. 3 do not happen when the rotation axis is slightly shifted relative to the taper waist. Indeed, they are an exception and come from a particular orientation of the elliptical cross section of the fiber in the taper waist relative to the rotation plane. Furthermore, the results obtained with the sensing configuration shown in Fig. 2(c) indicate the sensor sensitivity to the magnitude of the rotation angle but not to its sign (clockwise or anticlockwise rotation). In many situations, this information is enough, but there are others where the sign of the rotation is also required.

The taper fabrication with the electric-arc technique introduces a certain degree of azimuthal variation of the fiber radius in the taper waist. As a consequence of this change in fiber radius, different slopes appear for the variation of the fiber interferometer visibility with positive and negative rotation angles. Figure 4 illustrates the results that are normally obtained where the asymmetry behaviors for clockwise and anticlockwise rotations are evident.

It was mentioned before that the rotation angle does not introduce a phase variation in the fibermodal interferometer. This fact happens because the capillary tube that protects the fiber in the interferometer length extends up to the taper waist, which means all curvature associated with a particular rotation angle appears in this region. Such a situation is different if the capillary tube is moved slightly away from the taper along the interferometer length [a displacement to the right in Fig. 2(c)]. In this case,

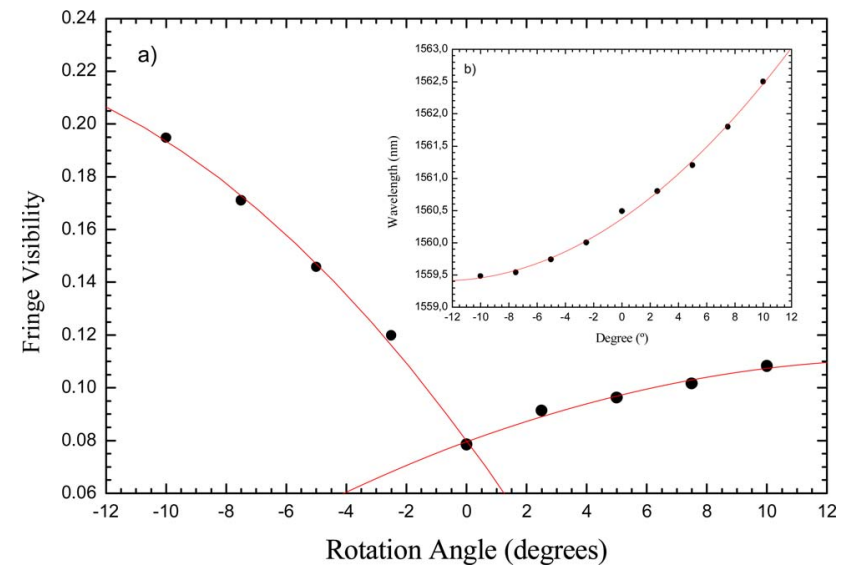

Fig. 4. (Color online) Visibility of the fiber-modal interferometer versus the rotation angle with a certain length of fiber not protected by the capillary tube. Inset: variation of the peak wavelength of the interferogram central fringe. for a certain extent, the curvature will also appear in the unprotected length of the fiber, introducing a differential delay in the fiber interferometer that will be a function of the rotation angle. If the orientation of the elliptical cross section of the fiber in the taper waist is properly chosen relative to the rotation plane, it will be possible, in principle, to have a monotonic variation of the interferometer phase with the rotation angle within a certain interval (even for positive and negative values). In fact, this possibility is presented in the inset of Fig. 4, where the monotonic shift of the peak wavelength of the interferometric fringe is related to the rotation angle changes between $-10^{\circ}$ and $+10^{\circ}$. Such a peak was initially located at the central region of the LPG spectral resonance. It is interesting to note that the $3 \mathrm{~nm}$ variation of the channeled spectrum in this rotation range corresponds to an interferometric phase variation of $\sim 90^{\circ}$. Moreover, the two graphs in Fig. 4 were obtained under the same experimental conditions where the unprotected length of the fiber interferometer was $\sim 5 \mathrm{~mm}$.

These results indicate that with the sensing configuration shown in Fig. 2(c) it is possible to measure not only the magnitude of the rotation angle but also its sign. While the magnitude measurement can be performed either by measuring the interferometer phase or by measuring the interferometer visibility, the magnitude and the sign determinations need both parameters, since it is necessary to have some additional information to overcome the sign ambiguity. The selection of one of these approaches will essentially depend on the particular constraints associated with each application.

To summarize, in this work, a novel concept was presented to implement a fiber-modal interferometer based on the combination of a fused taper and a longperiod grating. It was demonstrated that such a structure can be effectively applied to measure the rotation angle magnitude and to obtain, under certain conditions, the rotation sign.

O. Frazão's e-mail address is ofrazao @inescporto.pt.

\section{References}

1. A. M. Vengsarkar, P. J. Lemaire, J. B. Judkins, V. Bhatia, T. Erdogan, and J. E. Sipe, J. Lightwave Technol. 14, 58 (1996).

2. H. Ke, K. S. Chiang, and J. H. Peng, IEEE Photon. Technol. Lett. 10, 1596 (1998).

3. Y. Liu, J. A. R. Williams, L. Zhang, and I. Bennion, Opt. Commun. 164, 27 (1999).

4. X. J. Gu, Opt. Lett. 23, 509 (1998).

5. Y. G. Han, B. H. Lee, W. T. Han, U. C. Paek, and Y. Chung, Meas. Sci. Technol. 12, 778 (2001).

6. B. H. Lee and J. Nishii, Electron. Lett. 34, 2059 (1998).

7. P. L. Swart, Meas. Sci. Technol. 15, 1576 (2004).

8. E. H. Hecht, Optics (Addison-Wesley, 1987).

9. X. Dong, L. Su, P. Shum, Y. Chung, and C. C. Chan, Opt. Commun. 258, 159 (2005).

10. G. Rego, O. Okhotnikov, E. Dianov, and V. Sulimov, J. Lightwave Technol. 19, 1574 (2001). 